\title{
Importancia de algunas frutas, verduras y hortalizas en la prevención de la diabetes mellitus tipo II
}

The Importance of some fruits and vegetables in the prevention of diabetes mellitus type II

\author{
Marisela Martínez-Gamboa \\ Universidad Autónoma de Baja California \\ marisela.martinez@uabc.edu.mx \\ María de los Remedios Sánchez-Díaz \\ Universidad Autónoma de Baja California \\ maria.sanchez@uabc.edu.mx
}

\section{Resumen}

Los vegetales y frutas son extremadamente importantes en la nutrición humana como fuente de nutrientes y para la reducción de riesgo a desarrollar ciertas enfermedades crónicas.

Dentro de este grupo de enfermedades se encuentra la diabetes mellitus, la cual es una enfermedad de origen multifactorial, caracterizada por un grupo de desórdenes metabólicos que dan origen a la hiperglicemia crónica, uno de los síntomas característicos de esta enfermedad.

Históricamente, para el tratamiento se han empleado diversos medicamentos, pero recientemente se ha hecho evidente la importancia de la dieta en el control y la prevención de esta enfermedad. 
Son varias las especies vegetales recomendadas por la medicina tradicional que forman parte del consumo común en la dieta de la población mexicana y que además de sus efectos hipoglucemiantes, son ricas en vitaminas y minerales necesarios para una nutrición adecuada.

En este trabajo se discutirán las propiedades de algunos de estos alimentos que les permiten tener un efecto en la disminución de la concentración de glucosa sanguínea.

Palabras clave: Diabetes mellitus, hipoglucemiantes, hiperglicemia, plantas medicinales, índice glicémico.

\section{Abstract}

Vegetables and fruits are extremely important in human nutrition as a source of nutrients and to reduce risk of developing certain chronic diseases.

Within this group of diseases include diabetes mellitus, which is a disease of multifactorial origin, characterized by a group of metabolic disorders that give rise to chronic hyperglycemia, one of the characteristic symptoms of this disease.

Key Words: Diabetes mellitus, hypoglycemic, hyperglycemia, medicinal plants, glycemic index.

Fecha recepción: Septiembre 2012

Fecha aceptación: Noviembre 2012

\section{Introducción}

La diabetes mellitus es una enfermedad clasificada dentro del grupo de las crónicodegenerativas, es de origen multifactorial y caracterizada por un grupo de desórdenes metabólicos como la hiperglicemia crónica, la cual es causada por una deficiencia absoluta 
o relativa de insulina, la hormona clave en el mantenimiento de la normoglicemia (Sjöholm, 1996), y que es producida exclusivamente por las células beta del páncreas.

En el mundo, hay más de 347 millones de personas con diabetes y según datos de la Organización Mundial de la Salud (OMS) se calcula que en 2008 fallecieron 1.3 millones de personas.

Particularmente para la región de América del Norte y el Caribe, la Federación Internacional de Diabetes en 2012, nos indica que la prevalencia de esta enfermedad fue de $10.5 \%$, es decir, 1 de cada 10 adultos en esta región tiene diabetes.

Históricamente, para el tratamiento se han empleado diversos medicamentos, pero recientemente se ha hecho evidente la importancia de la dieta en el control y la prevención de esta enfermedad.

Son varias las especies vegetales recomendadas por la medicina tradicional que forman parte del consumo común en la dieta de la población mexicana y que además de sus efectos hipoglucemiantes, son ricas en vitaminas y minerales necesarios para una nutrición adecuada.

El propósito de este trabajo es describir los efectos de algunos de estos vegetales de consumo común en la dieta de la población mexicana, y su papel en la prevención de la diabetes mellitus.

\section{SECRECIÓN DE INSULINA Y DIABETES}

Las células endocrinas están organizadas en microorganos altamente vascularizados e inervados esparcidos por todo el páncreas llamados islotes de Langerhans (Guz, Nasir \& Teitelman, 2001). El 60 a $80 \%$ de estas estructuras lo componen las células $\beta$, que se encargan de producir insulina, la única hormona en el cuerpo que reduce la glucosa sanguínea. 
La integración del mecanismo dentro de las células beta en los islotes de Langerhans es responsable de la liberación de la insulina en respuesta al estímulo con glucosa, y por su parte la insulina estimula la utilización de glucosa en los tejidos periféricos como músculo esquelético e hígado. Cualquier alteración en este mecanismo puede llevar a desarrollar intolerancia a la glucosa o diabetes mellitus (Herchuelz, Diaz-Horta \& Van Heylen, 2002; Navarro-Tableros, Fiordelisio, Hernández-Cruz \& Hiriart, 2007).

La diabetes mellitus tipo II (no insulino-dependiente), se caracteriza por la resistencia a la insulina (Edlund, 2001). Esta enfermedad se considera resultado de la combinación de varios factores como la predisposición genética, factores ambientales que incluyen la nutrición y el ejercicio físico. (Rossini, Mordes, Greiner \& Stoff, 2001). Es la condición con más incidencia en individuos de edad mediana o avanzada, de los cuales el $80 \%$ son obesos.

\section{FIBRA DIETÉTICA Y METABOLISMO DE GLUCOSA}

La fibra dietetica es un grupo de sustancias complejas que pueden ser descritas como carbohidratos no digeribles y ligninas que no se degradan en el tracto gastrointestinal superior (Weickerth \& Pfeiffer, 2008), la podemos encontrar en verduras, frutas, cereales, granos y semillas. Se recomienda que una buena nutrición incluye una ingesta diaria de 20 a $40 \mathrm{~g}$ de fibra.

La falta de fibra dietética puede ser un factor desencadenante de la diabetes mellitus tipo II (Montonen, Knekt, Järvinen, Aromaa, \& Reunanen, 2003), investigaciones recientes indican que el consumo de fibra dietética contribuye a una serie de efectos metabólicos inesperados independientes de cambios en el peso corporal, los cuales incluyen aumento a la sensibilidad a insulina (Gary et al., 2004), modulación de algunas hormonas 
intestinales, y efectos en varios marcadores metabólicos e inflamatorios asociados con el síndrome metabólico (Steyn et al., 2004).

El contenido de fibra dietética soluble ha mostrado darle a los alimentos textura y viscosidad, hecho que influye en la inhibición de la absorción de macronutrientes como el almidón, regula los niveles de glucosa sanguínea posprandial e influye beneficiosamente en algunos lípidos sanguíneos. (Brennan, 2005).

ÍNDICE Y CARGA GLICÉMICA: CARBOHIDRATOS PROVENIENTES DE LA DIETA

Dentro de la prevención de la diabetes, la dieta juega un papel determinante (WHO, 2003). La calidad de los carbohidratos ingeridos es de extrema importancia para determinar la habilidad de estas moléculas para aumentar los niveles de glucosa, lo que depende en gran medida en su influencia en el transito gastrointestinal y la velocidad de absorción de nutrientes y el riesgo a desarrollar diabetes (Salas-Salvadó, MartínezGonzález, Bulló \& Ros, 2011).

Un método útil para clasificar a los alimentos con base en el impacto de los hidratos de carbono disponibles en el alimento es el índice glucémico (Jenkins, A. L., Jenkins, D. J., Zdravkovic, Wursch, Vuksan, 2002).

Los índices glicémicos se determinan en valores de glucosa posprandial y son una herramienta útil en la determinación de la velocidad a la que los carbohidratos de la dieta se digieren y se absorben como glucosa. Individuos que requieren un nivel alto de glucosa en sangre inmediatamente después de la ingestión deben seleccionar alimentos con índice glucémico alto. 
Los datos registrados en la Tabla 1 indican que la fuente de carbohidratos tienen un papel importante en el impacto glucémico global de la alimentación, a menudo valores de índice glicémico alto se relacionan con el aumento de peso y algunos consumidores pretenden evitar la ingesta de carbohidratos y sustituirla con alimentos ricos en proteínas o grasas.

Tabla 1. Indice glicémico y de carga glicémica de algunos alimentos consumidos en México (Noriega, 2004).

\begin{tabular}{|l|l|l|}
\hline ALIMENTO & IG & CG \\
\hline Tortilla de harina de trigo & 30 & 8 \\
\hline Tortillla de maíz & 52 & 12 \\
\hline Pan dulce & 67 & 17 \\
\hline Pan integral & 71 & 9 \\
\hline Arroz & $55-64$ & $18-23$ \\
\hline Camote & 61 & 17 \\
\hline Papas & $74-92$ & $15-26$ \\
\hline Frijoles pintos & $14-18$ & $1-4$ \\
\hline Frijoles negros & 30 & 7 \\
\hline Frijoles bayos & 38 & 12 \\
\hline Garbanzos & 28 & 8 \\
\hline Lentejas & 29 & 5 \\
\hline Alubias & 38 & 12 \\
\hline Chícharos & 48 & 3 \\
\hline Nopales & 7 & 0 \\
\hline Zanahoria & 53 & 5 \\
\hline Elote & 75 & 17 \\
\hline Calabaza & & 3 \\
\hline
\end{tabular}




\begin{tabular}{|l|l|l|}
\hline Manzana & 38 & 6 \\
\hline Pera & 38 & 4 \\
\hline Fresa & 40 & 1 \\
\hline Naranja & 42 & 5 \\
\hline Durazno & 42 & 5 \\
\hline Uvas & 46 & 8 \\
\hline Mango & 51 & 8 \\
\hline Plátano & 52 & 12 \\
\hline Papaya & 59 & 10 \\
\hline Piña & 59 & 7 \\
\hline Melón & 65 & 4 \\
\hline Sandía & 72 & 4 \\
\hline Dátiles & 103 & 42 \\
\hline
\end{tabular}

IG, índice glicémico. CG, carga glicémica.

IG bajo (lento) $\leq 55$; IG moderado 56-69; IG alto (rápido) $\geq 70$

Los alimentos con índice glicémico bajos son útiles para consumidores que deseen disminuir la disponibilidad de glucosa con la finalidad de mantener o reducir el peso (Jimenez-Cruz, Seimandi \& Bacardi-Gascón, 2003), pero la respuesta total a la glucosa sanguínea esta determinada no solamente por el índice glucémico de una comida, sino por la cantidad de carbohidratos (Hu, van Dam \& Liu 2001), para lo cual se ha desarrollado el termino carga glicémica (CG), el cual difiere del índice glicémico en que la carga glicémica se refiere al impacto que el total de los alimentos consumidos en la dieta tienen en la producción de glucosa, se toma en cuenta la cantidad relativa de carbohidratos (Torres, Palacios-González, Noriega-López \& Tovar-Palacio, 2004).

\section{LA DIETA MEXICANA Y DIABETES MELLITUS}

La diabetes es el principal problema de salud en México, de acuerdo a datos arrojados por el Instituto Mexicano del Seguro Social (IMSS), en 2009 se registraron un promedio diario 
de 477 casos, posicionando al país en el sexto lugar a nivel mundial, con más de 6.4 millones de casos.

Aunque el estilo de vida es el principal responsable de los altos niveles de obesidad y diabetes entre los mexicanos, también hay otras consideraciones importantes como la herencia de genes ahorrativos, indicando que el riesgo de padecer diabetes es un resultado de los mecanismos intergeneracionales (Martorell, 2005).

La dieta mexicana es rica en hidratos de carbono, en un estudio reciente se encontró que la cantidad de carbohidratos presentes fue de 75 al $80 \%$ de las calorías totales ingeridas, basada principalmente de almidón. Sólo el $6 \%$ de las calorías totales derivaron de azúcares simples, el consumo de fibra cruda fue alto, de 18 a 21 g por día.

La prevención de la diabetes a través de la inclusión de alimentos ricos en carbohidratos complejos y fibra dietetica es la forma más plausible para modificar el crecimiento de la epidemia (Aguilar-Salinas \& Gómez-Pérez, 2006). Un ejemplo de esto es la dieta de los indios Tarahumara, compuesta principalmente por frijol y maíz, que proporcionan un alto consumo de carbohidratos complejos, es baja en grasa y colesterol, e indica alta calidad nutricional y es considerada antiaterogénica (Cerqueira, M.T., McFurry, F.M. \& Connor, W.E., 1979).

PLANTAS COMESTIBLES PRESENTES EN LA DIETA MEXICANA Y LA PREVENCION DE DIABETES MELLITUS TIPO ॥

Algunas plantas y vegetales usadas por la población mexicana para la prevención y tratamiento de la diabetes mellitus tipo II son infusiones orales tomadas durante el día o también son frutas y verduras como se resume en la Tabla 2. Este hecho tiene una importancia peculiar ya que va ligado a dos factores básicos para el control de la DM: alimentación y medicación. Un menú que incluya este tipo de plantas comestibles podría de alguna forma en los pacientes diabéticos a mejorar su dieta y de esta manera controlar su enfermedad reduciendo la dosis de los fármacos hipoglicémicos (Alarcón-Aguilar et al., 1995; Andrade-Cetto et al., 2004; Revilla-Monsalve et al., 2007). 
Tabla 2. Plantas comestibles y frutas estudiadas en el tratamiento tradicional de la DM. ${ }^{a}$

\begin{tabular}{|l|l|l|l|}
\hline Nombre cientifico & Nombre común & Estructura & Preparacion $^{b}$ \\
\hline $\begin{array}{l}\text { Allium cepa L. } \\
\text { (Liliaceae) }\end{array}$ & Cebolla & Bulbo & $\begin{array}{l}\text { Decocción, } \\
\text { cruda }\end{array}$ \\
\hline $\begin{array}{l}\text { Allium sativum L. } \\
\text { (Liliaceae) }\end{array}$ & Ajo & Bulbo & Decocción \\
\hline $\begin{array}{l}\text { Ananas comosus } \\
\text { (L.) } \\
\text { (Bromeliaceae) }\end{array}$ & Piña & Fruta & Juice \\
\hline $\begin{array}{l}\text { Annona glabra L. } \\
\text { (Annonaceae) }\end{array}$ & Anona silvestre & Fruta & \\
\hline $\begin{array}{l}\text { Annona muricata L. } \\
\text { (Annonaceae) }\end{array}$ & Guanabana & Fruta & Jugo, infusión \\
\hline $\begin{array}{l}\text { Brassica oleracea L. } \\
\text { (Cruciferae) }\end{array}$ & Col de brusselas & Hojas & Juda \\
\hline $\begin{array}{l}\text { Brassica oleracea L. } \\
\text { var. } \\
\text { (Cruciferae) }\end{array}$ & Coliflor \\
(Myrsonima & Frassifolia & Inflorescencia & Jugo \\
\hline $\begin{array}{l}\text { Citrus aurantifolia } \\
\text { (Munth. }\end{array}$ & Limón & Jugo \\
\hline
\end{tabular}




\begin{tabular}{|c|c|c|c|}
\hline $\begin{array}{l}\text { (Christm.) Swingle } \\
\text { (Rutaceae) }\end{array}$ & & & \\
\hline $\begin{array}{l}\text { Citrus limetta Risso } \\
\text { (Rutaceae ) }\end{array}$ & Lima & Fruta & Fruit \\
\hline $\begin{array}{l}\text { Coriandrum } \\
\text { sativum } \\
\text { (Apiaceae) }\end{array}$ & Cilantro & Partes aereas & infusión \\
\hline $\begin{array}{l}\text { Cucumis sativus L. } \\
\text { (Cucurbitaceae) }\end{array}$ & Calabaza & Fruta & Jugo \\
\hline $\begin{array}{l}\text { Cucurbita ficifolia } \\
\text { Bouche } \\
\text { (Cucurbitaceae) }\end{array}$ & Calabaza & Fruta & Jugo \\
\hline $\begin{array}{l}\text { Cucurbita maxima } \\
\text { Duchesne } \\
\text { (Cucurbitaceae) }\end{array}$ & Calabaza & Fruta & Jugo \\
\hline $\begin{array}{l}\text { Cucurbita mexicana } \\
\text { Damm } \\
\text { (Cucurbitaceae) }\end{array}$ & Calabaza, Melón & Hojas y fruta & $\begin{array}{l}\text { infusión } \quad y \\
\text { jugo }\end{array}$ \\
\hline $\begin{array}{l}\text { Daucus carota L. } \\
\text { (Apiaceae) }\end{array}$ & Zanahoria & Raíz & Jugo \\
\hline $\begin{array}{l}\text { Eucalyptus globules } \\
\text { Labill. (Myrtaceae) }\end{array}$ & Eucalipto & Hojas & infusión \\
\hline Nombre cientifico & Nombre común & Estructura & Preparacion $^{b}$ \\
\hline $\begin{array}{l}\text { Lactuca sativa L. } \\
\text { var. Romana. } \\
\text { (Compositae) }\end{array}$ & Lechuga romana & Hojas & Jugo \\
\hline $\begin{array}{l}\text { Mentha piperita L. } \\
\text { Lamiaceae }\end{array}$ & Hierbabuena & Hojas & infusión \\
\hline
\end{tabular}




\begin{tabular}{|c|c|c|c|}
\hline $\begin{array}{l}\text { Nasturtium } \\
\text { officinale R. Br. } \\
\text { (Brassicaeae) }\end{array}$ & Berro & Partes aereas & infusión \\
\hline $\begin{array}{l}\text { Nopalea } \\
\text { cochenillifera (L.) } \\
\text { Salm-Dyck } \\
\text { (Cactaceae) }\end{array}$ & Nopal & Tallo & cruda \\
\hline $\begin{array}{l}\text { Opuntia ficus-indica } \\
\text { (L.) Mill. } \\
\text { (Cactaceae) }\end{array}$ & Nopal & Tallo & cruda \\
\hline $\begin{array}{l}\text { Opuntia imbricata } \\
\text { (Haw) DC. } \\
\text { (Cactaceae) }\end{array}$ & Xoconostle & Tallo y fruta & cruda \\
\hline $\begin{array}{l}\text { Opuntia } \\
\text { streptacantha } \\
\text { Lem. (Cactaceae) }\end{array}$ & Nopal & Tallo & cruda \\
\hline $\begin{array}{l}\text { Opuntia } \\
\text { streptacantha } \\
\text { Lemaire. } \\
\text { (Cactaceae) }\end{array}$ & Nopal & Corteza & Jugo \\
\hline $\begin{array}{l}\text { Petroselinum } \\
\text { crispum }\end{array}$ & Perejil & Partes aereas & infusión \\
\hline
\end{tabular}




\begin{tabular}{|c|c|c|c|}
\hline $\begin{array}{l}\text { (Mill.) Nyman ex } \\
\text { A.W. Hill. } \\
\text { (Apiaceae) }\end{array}$ & & & \\
\hline $\begin{array}{l}\text { Phaseolus vulgaris } \\
\text { L. (Fabaceae) }\end{array}$ & Frijol & Fruta & infusión \\
\hline $\begin{array}{l}\text { Physalis } \\
\text { philadelphica Lam. } \\
\text { (Solanaceae) }\end{array}$ & Tomate & Fruta & tostadas \\
\hline $\begin{array}{l}\text { Piper sanctum } \\
\text { (Miq.) } \\
\text { Schltdl. ex C. DC. } \\
\text { (Piperaceae) }\end{array}$ & Hierba Santa & Hojas & infusión \\
\hline $\begin{array}{l}\text { Portulaca denudata } \\
\text { Poelln. } \\
\text { (Portulacaceae) }\end{array}$ & Verdolaga & Partes aereas & Infusión \\
\hline Nombre cientifico & Nombre común & Estructura & Preparacion $^{b}$ \\
\hline $\begin{array}{l}\text { Portulaca oleracea } \\
\text { L. (Portulacaceae) }\end{array}$ & Verdolaga & Partes aereas & Infusión \\
\hline $\begin{array}{l}\text { Prunus serotina } \\
\text { subsp. capuli (Cav.) } \\
\text { McVaugh. } \\
\text { (Rosaceae) }\end{array}$ & Capulín & Fruta & Infusión \\
\hline $\begin{array}{lr}\text { Prosopis } & \text { juliflora } \\
\text { (Sw.) } & \text { DC. } \\
\text { (Fabaceae) } & \end{array}$ & Mezquite & Fruta & cruda \\
\hline Psidium guajava L. & Guayaba & Fruta & Cruda \\
\hline
\end{tabular}




\begin{tabular}{|l|l|l|l|}
\hline $\begin{array}{l}\text { (Myrtaceae) } \\
\text { Sechium Sw. } \\
\text { (Jacq.) } \\
\text { (Cucurbitaceae) }\end{array}$ & Chayote & Fruta & infusión \\
\hline $\begin{array}{l}\text { Solanum Wright. } \\
\text { verbascifolium }\end{array}$ & Berenjena & Partes aereas & Infusión \\
C.B. & & \\
(Solaneceae) & Hojas & Jugo \\
\hline $\begin{array}{l}\text { Spinacea oleracea } \\
\text { L. (Chenopodiceae) }\end{array}$ & Espinaca & Pruta & Cruda y pulpa \\
\hline $\begin{array}{l}\text { Tamarindus indica } \\
\text { L. (Fabaceae) }\end{array}$ & Tamarindo & Infusión \\
\hline $\begin{array}{l}\text { Zea mays } \\
\text { h.(Poaceae) }\end{array}$ & Maíz & & \\
\hline
\end{tabular}

a. En 1993 se depositó el voucher de clasificación en el Herbario del IMSS.

b. Decocción de 132 g de planta seca hervida en 1 L de agua/10 min. Infusión 12 a 15 g de planta seca a la que se agrega $1 \mathrm{~L}$ de agua hirviendo.

Dentro de la dieta mexicana los vegetales más consumidos y que poseen propiedades hipoglucemiantes son el maíz, frijol, tomate y cebolla (Martínez Jasso \& Villezca Becerra, 2003).

El fruto de la planta de frijol (Phaseolus vulgaris L.) es uno de los remedios tradicionales más utilizados contra la diabetes debido a su alto contenido en fibra y su efecto inhibitorio de la $\alpha$-amilasa, lo cual puede ser efectivo en la prevención o disminución de los niveles de glucosa en la diabetes mellitus tipo II (Helmsädter, 2010). 
Por su parte el tomate (Physalis philadelphica Lam.) además de ser una gran fuente de fibra, posee licopeno, el cual causa un decremento dosis-dependiente en los niveles de glucosa, un aumento de la concentración de insulina y un aumento de la actividad antioxidante de algunas enzimas (CAT, SOD y GPx), asi como una mejora en el perfil de lípidos séricos (Ali \& Agha, 2009)

La cebolla posee reconocidos efectos hipoglucemiantes, recientemente la S-metil cisteína sulfoxido (SMCS) un sulfuro que contiene aminoácidos aislados de la cebolla, mostró efectos antidiabéticos y antihiperlipidémicos controlando los niveles de glucosa en sangre y lípidos en suero y tejidos alterando la actividad de la hexoquinasas hepática, glucosa-6fosfatasa HMG CoA reductasa a niveles normales, mostrando efectos comparables a la glibenclamida e insulina (Kumari \& Augusti, 2002).

Los inhibidores de la aldosa reductasa $(A R)$ tienen un potencial terapéutico considerable contra las complicaciones diabéticas y no aumentan el riesgo de hipoglucemia, este hecho ha sido demostrado por Tae Hyeon Kim et al en (2013) realizando experimentos con productos aislados del maíz morado (Zea mays L.), en donde uno de los 12 compuestos aislados, la hersutrina demostró tener una potente capacidad inhibidora de actividad AR, además de inhibir la reducción de galactosa a galactitol en lente y eritrocitos de rata, lo cual indica que la hersutrina efectivamente puede prevenir el estrés osmótico en estado de hiperglicemia, pudiendo así decir que la hersutrina, derivada del maíz morado puede ser un agente terapéutico potencial contra complicaciones de la diabetes.

\section{Conclusión}

De acuerdo a la información presentada, una dieta que incluye el consumo adecuado de frutas, verduras y hortalizas es de suma importancia para la disponibilidad de fibra dietética, uno de los factores mas importantes en la prevención de la diabetes mellitus. 
Las plantas utilizadas en la medicina tradicional mexicana tienen evidentes efectos hipoglucemiantes y su utilización puede tener efectos beneficiosos en pacientes prediabéticos o en pacientes diabéticos podrían ayudar a tener un mejor control de los niveles de glucosa y la utilización de insulina.

\section{Bibliografía}

Aguilar-Salinas, C.A., Gómez-Pérez, F.J. (2006). Declaración de Acapulco: propuesta para la reducción de la incidencia de la diabetes en México, Rev. invest. clín, 58(1), 71-77.

Alarcón-Aguilar, F.J. Roman-Ramos, R. \& Flores-Saenz, J. L. (2005). Plantas medicinales usadas en el control de la diabetes mellitus, Ciencia, 44, 363-371.

Ali, M. M. \& Agha, F.G. (2009). Amelioration of streptozotocin-induced diabetes mellitus, oxidative stress and dyslipidemia in rats by tomato extract lycopene, Scandinavian Journal of Clinical \& Laboratory Investigation, 69(3), 371-379.

Andrade-Cetto, A. \& Wiedenfeld, M. (2004). Hypoglycemic effect of Acosmiun panamense bark on streptozotocin diabetic rats, Journal of Ethnopharmacology, 90, 217-220.

Brennan, C.S. (2005). Dietary fiber, glycaemic response, and diabetes. Mol. Nutr. Food Res, $49,560-570$.

Cerqueira, M.T., McMurry, F.M. \& Connor W.E. (1979). The food and nutrient intakes of the Tarahumara Indians of Mexico, International Nutrition, Am J Clin Nutrition, 32, 905915.

Edlund, H. (2001). Factors controlling pancreatic cell differentiation and function, Diabetologia, vol.44 pp.1071-1079. 
Gary, T. L., Baptiste-Roberts, K., Gregg, E. W., Williams, D. E., Beckles, G.L.A., Miller, E. J., Engelgau, M. M., (2004). Fruit, Vegetable and Fat Intake in a Population-Based Sample of African Americans. Journal of the National Medical Association, 96(12), 1599-1605.

Guz, Y., Nasir, I. \& Teitelman G. (2001), Regeneration of pancreatic $\beta$ cells from intra-islet precursor cells in an experimental model of diabetes, Endocrinology, 142, 4956-4968.

Jenkins, A. L., Jenkins, D. J. A., Zdravkovic, U., Wursch, P. \& Vuksan, V. (2002) Depression of the glycaemic index by high levels of beta-glucan fibre in two functional foods tested in type 2 diabetes. Eur. J. Clin. Nutr, 56, 622-628.

Kim T.H., Kim, J.K., Kang, Y.H., Lee, J.Y., Kang, J. \& Lim, S.S. (2013) Aldose Reductase Inhibitory Activity of Compounds from Zea mays L., BioMed Research International, 1-8.

Montonen, J., Knekt, P., Järvinen, R., Aromaa, A., \& Reunanen, A. (2003). Whole-grain and fiber intake and the incidence of type 2 diabetes. Am J Clin Nutr, 77, 622-9.

Navarro-Tableros, V. Fiordelisio, T., Hernández-Cruz, A. \& Hiriart, M. (2007), Physiological development of insulin secretion, calcium channels, and GLUT2 expression of pancreatic rat beta cell, American Journal of Physiology, Endocrinology and metabolism, 292, 1018-1029.

Revilla-Monsalve, M. C., Andrade-Cetto, A., Palomino-Garibay, A., Wiedenfeld, H. \& IslasAndrade, S. (2007), Hipoglycemic effect of Cecropia obtusifolia Bertol aqueous extracts on type 2 diabetic patients, Journal of Ethnopharmacology, 111, 636-640.

Rossini A., Mordes, J.P., Greiner, D.L. \& Stoff J.S. (2001), Islet cell transplantation tolerance, Transplantation, 72(8), 43-46.

Steyn, N.P., Mann, J., Bennett, P.H., Temple, N., Zimmet, P., Tuomilehto, J., Lindström, J., Louheranta, A. (2004), Diet, nutrition and the prevention of type 2 diabetes, Public Health Nutrition, 7(1A), 147-165.

Torres, N., Palacios-González, M., Noriega-López, L. \& Tovar-Palacio, A.R., (2006), Indice 
glicémico, índice insulinémico y carga glicémica de bebidas de soya con un contenido bajo y alto en hidratos de carbono, Rev Inv Clin, 58(5), 487-497.

Weickert, M.O. \& Pfeiffer, A.F.H. (2008), Metabolic Effects of Dietary Fiber Consumption and Prevention of Diabetes, American Society for Nutrition, J. Nutr, 138, 439-442. 